\title{
Recalling Routes around London: Activation of the Right Hippocampus in Taxi Drivers
}

\author{
Eleanor A. Maguire, Richard S. J. Frackowiak, and Christopher D. Frith \\ Wellcome Department of Cognitive Neurology, Institute of Neurology, London WC1N 3BG, United Kingdom
}

Functional imaging to date has examined the neural basis of knowledge of spatial layouts of large-scale environments typically in the context of episodic memory with specific spatiotemporal references. Much human behavior, however, takes place in very familiar environments in which knowledge of spatial layouts has entered the domain of general facts often referred to as semantic memory. In this study, positron emission tomography (PET) was used to examine the neural substrates of topographical memory retrieval in licensed London taxi drivers of many years experience while they recalled complex routes around the city. Compared with baseline and other nontopographical memory tasks, this resulted in activation of a network of brain regions, including the right hippocampus. Recall of famous landmarks for which subjects had no knowl- edge of their location within a spatial framework activated similar regions, except for the right hippocampus. This suggests that the hippocampus is involved in the processing of spatial layouts established over long time courses. The involvement of similar brain areas in routes and landmarks memory indicates that the topographical memory system may be primed to respond to any relevant topographical stimulation; however, the right hippocampus is recruited specifically for navigation in large-scale spatial environments. In contrast, nontopographical semantic memory retrieval involved the left inferior frontal gyrus, with no change in activity in medial temporal regions.

Key words: PET; topographical memory; landmarks; taxi drivers; semantic memory; hippocampus
Several functional imaging studies document involvement of the human hippocampal formation/parahippocampal gyrus in acquisition (Aguirre et al., 1996; Maguire et al., 1996a, 1997) and retrieval (Aguirre et al., 1996; Ghaem et al., 1996) of knowledge of large-scale environmental space. These studies used tests of episodic memory in which topographical learning had a specific spatiotemporal reference (Tulving, 1983) and in which such memory retrieval may still have involved a degree of active encoding of the environment. Undoubtedly knowledge of environmental space has its basis in distinct episodes of learning; however, much human behavior takes place in environments with which we are very familiar, and knowledge of their spatial layout has entered the domain of general facts about the world, often referred to as semantic memory. The neural substrates of topographical memories of long standing (i.e., over several years) have not yet been examined with functional imaging, although it is known from case studies of patients with topographical disorientation in familiar environments that such memories can be impaired (Landis et al., 1986; Habib and Sirigu, 1987). Previous functional imaging work indicates that there may be different anatomical substrates for verbal episodic and semantic memory retrieval (Fletcher et al., 1995b; Tulving et al., 1996). The aim of the present study was to examine semantic topographical memory retrieval and to determine whether recall of well established spatial layouts activates similar brain regions noted in other studies to subserve episodic topographical memory, with reference in particular to the medial temporal region.

Received April 10, 1997; revised July 7, 1997; accepted July 9, 1997.

This work was supported by The Wellcome Trust.

Correspondence should be addressed to Dr. Eleanor A. Maguire, Wellcome Department of Cognitive Neurology, Institute of Neurology, 12 Queen Square, London WC1N 3BG, UK.

Copyright (C) 1997 Society for Neuroscience $\quad 0270-6474 / 97 / 177103-08 \$ 05.00 / 0$
Topographical knowledge is thought to comprise information about both landmarks and spatial relations between landmarks (Siegal and White, 1975; Thorndyke and Hayes, 1982). Evidence from patient studies of different anatomical substrates for landmark and location knowledge is inconsistent. Cases of topographical disorientation in which the main deficit was spatial and not an agnosia for landmarks have been reported after lesions to posterior parietal cortex (DeRenzi et al., 1977); however, patients with topographical learning deficits of a similar spatial character after circumscribed medial temporal lesions have also been reported (Maguire et al., 1996b). The current study set out to assess the neural instantiation of landmark knowledge, where such knowledge was not confounded by location information about position within a large-scale spatial layout. This was achieved by using a task in which famous landmarks were known but their large-scale spatial context was not. A final aim of the study was to examine topographical memory (landmarks/spatial layouts) and also nontopographic semantic memory retrieval to ascertain whether common brain regions subserve semantic memory regardless of memory type.

To examine well established topographical memory, we used subjects who were all licensed London taxi drivers of many years experience. Official London taxi drivers must train for $\sim 3$ years and pass stringent examinations of spatial knowledge before receiving a license. Their extensive knowledge of London meant that all subjects could be tested on the same stimuli, with high levels of retrieval success and with no encoding of new environmental information during task performance. Studies from environmental psychology indicate that experienced taxi drivers differ with respect to novices and the general public in their increased knowledge of shortcuts and minor streets, but not in the cognitive strategies used in way-finding (Chase, 1983; Pailhous, 1984; Peruch et al., 1989; Giraudo and Peruch, 1988). 


\section{MATERIALS AND METHODS}

Subjects. Eleven right-handed qualified and licensed male London taxi drivers (mean age $45 \pm 7$ years) participated in the study. None had a previous history of psychiatric or neurological illness. The average time spent working as a licensed London taxi driver was $14.55 \pm 12$ years, with the shortest time being 3 years. All subjects gave informed written consent, and the study was approved by the local hospital ethics committee and conducted under certification from the Administration of Radioactive Substances Advisory Committee (Department of Health, London, UK).

Scanning techniques. PET scans were obtained using a Siemens/CPS ECAT EXACT HR+ (model 962) PET scanner. Scanning was performed with septa retracted, in three-dimension (3-D) mode. The field of view of $15.5 \mathrm{~cm}$ in the axial extent allowed the whole brain to be studied simultaneously. Volunteers received an $\mathrm{H}_{2}{ }^{15} \mathrm{O}$ intravenous bolus (330 $\mathrm{MBq}$ ) infused over $20 \mathrm{sec}$ followed by a $20 \mathrm{sec}$ saline flush through a forearm cannula. The scan protocol included a delay frame of $15 \mathrm{sec}$ to monitor the average radioactivity counts in the scanner. The system allows the activation frame to be triggered automatically, depending on the physiology of the individual subject. Data were acquired in a $90 \mathrm{sec}$ scan frame. There were 12 successive administrations of $\mathrm{H}_{2}{ }^{15} \mathrm{O}$, each separated by $8 \mathrm{~min}$. The integrated radioactivity counts accumulated over the $90 \mathrm{sec}$ acquisition period, corrected for background, were used as an index of regional cerebral blood flow (rCBF). Attenuation correction was computed using a transmission scan before emission scan acquisition. Images were reconstructed into $128 \times 128$ pixels in 63 planes, with an in-plane resolution of $6.5 \mathrm{~mm}$. In addition, high resolution magnetic resonance imaging (MRI) scans were obtained with a 2.0 Tesla Vision system (Siemens GmbH, Erlangen, Germany) using a T1weighted 3-D gradient echo sequence. The image dimensions were $256 \times$ $256 \times 256$ voxels. The voxel size was $1 \times 1 \times 3 \mathrm{~mm}$.

Data analysis. Images were analyzed using Statistical Parametric Mapping (SPM96; Wellcome Department of Cognitive Neurology, London, UK: http://www.fil.ion.ucl.ac.uk/SPM) executed in MATLAB (Mathworks Inc., Sherborn MA). This approach combines the general linear model and the theory of Gaussian fields to make statistical inferences about regional blood flow effects (Friston et al., 1991, 1994; Worsley et al., 1992). All scans were automatically realigned to the first scan and then normalized using a nonlinear deformation (Friston et al., 1995) into standard stereotactic space (Talairach and Tournoux, 1988) using a template from the Montreal Neurological Institute (Evans et al., 1993). The structural MRI scans were normalized into the same space to allow for the superimposition of PET activations onto an averaged structural image. Images were smoothed using an isotropic Gaussian kernel of 16 $\mathrm{mm}$ (full width half maximum) to optimize the signal-to-noise ratio and to adjust for intersubject differences in gyral anatomy. Global variance between conditions was removed using analysis of covariance. For each pixel in stereotactic space, condition-specific adjusted rCBF values with an associated adjusted error variance were generated. Areas of significant change in brain activity were then determined using appropriately weighted contrasts between the task-specific scans and the $t$ statistic. The resulting sets of $t$ values constituted the statistical parametric map. Significance levels were set at $p<0.001$ (uncorrected).

Experimental tasks. There were six tasks, each performed twice, with task order counterbalanced within and between subjects; five of the tasks are relevant to this discussion. The study had a factorial design with two factors of interest. Figure 1 illustrates the experimental design. Two of the tasks involved topographical knowledge: in one, taxi drivers were given a starting and destination points in the greater London area. They had to describe overtly during scan acquisition the shortest legal route between the start and destinations (assuming normal traffic flow outside rush hour). The other topographical task involved recalling and describing the appearance of individual world-famous landmarks (not in London) that subjects had never visited. An important feature of navigation involves recalling information in a set sequence, whereas landmark knowledge can be recalled in any order. Another factor of interest in the design, therefore, was the requirement-or not-for sequencing. Two nontopographic tasks were also included to engage subjects in semantic memory retrieval in a manner similar to the topographical tasks, but without involving the recall of environmental spatial information. The nontopographical tasks concerned memory for films: recalling and describing the plots of familiar famous films between given points in the story line, which like the recall of routes involves a set sequence of information retrieval; and recalling and describing individual frames from famous films, not the plot of the film, which does not involve any

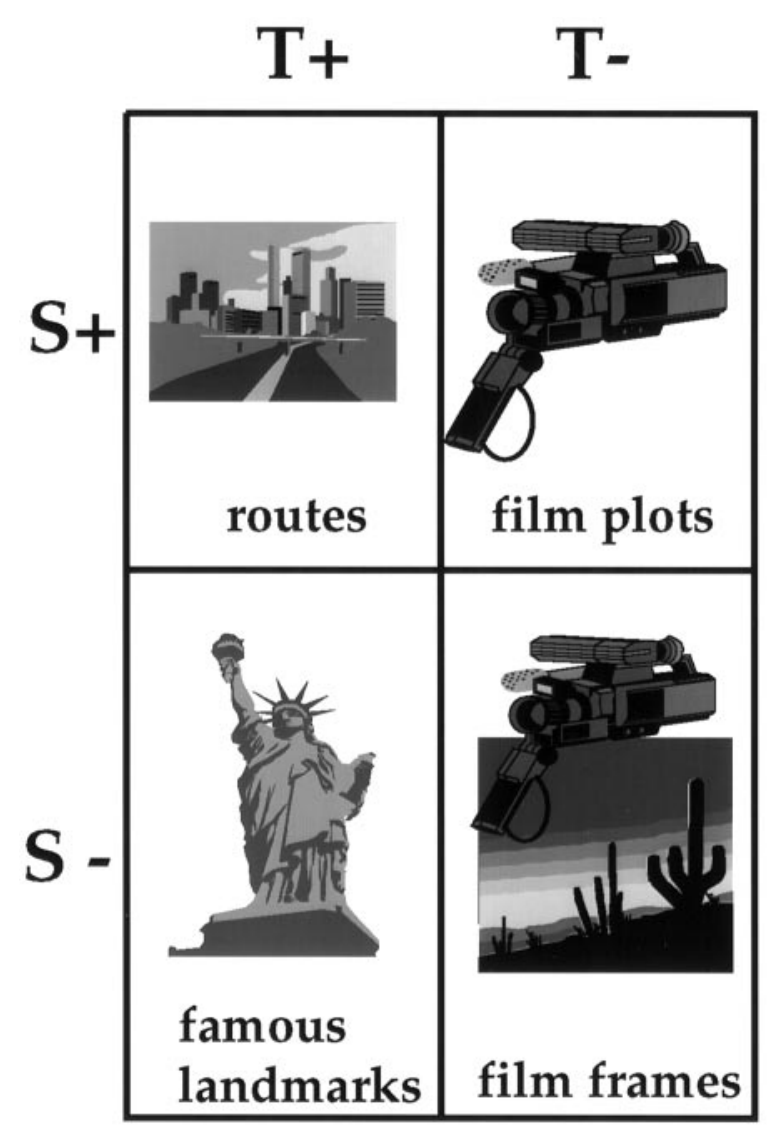

\section{number repetition baseline}

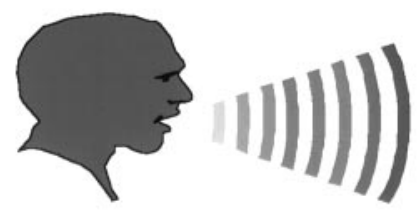

Figure 1. Experimental design, two factors of interest: T, topographical memory; $S$, sequencing. A baseline task was also included.

necessary sequence of information. Tasks, therefore, involved either topographical or nontopographical knowledge and had either a sequential component or not. A baseline task to control for speech output was included in which subjects repeated two four-digit numbers during scanning. Comparison with this baseline facilitated examination of the network of brain regions involved in each task by indicating whether changes of activity were relative or absolute in relation to it. Subjects were blindfolded throughout, and speech output was digitally recorded.

Before they arrived for scanning, subjects completed and returned questionnaires that inquired about the following: (1) areas of London with which they were most familiar; (2) films they would rate as very familiar, from a list of 150 color films made between 1939 and the present day; and (3) individual landmarks from a list of 20 world-famous ones they had visited in person and could visualize in their mind's eye. Routes from areas of London with which all subjects were familiar were chosen as stimuli in the routes recall task. Those films that all subjects selected in common as being very well known were used as stimuli in the film plots and film frames tasks. These were films that subjects were thoroughly familiar with and had generally seen five or more times, with no memory for where, when, or with whom they had seen the film (thus no spatiotemporal reference and therefore semantic memories). Famous land- 


\begin{tabular}{|c|c|c|c|}
\hline Task & Mean & SD & Range \\
\hline Routes & 55.62 & 11.08 & $36-75$ \\
\hline Landmarks & 58.23 & 7.76 & $43-70$ \\
\hline Film plots & 58.14 & 7.92 & $43-70$ \\
\hline Film frames & 56.36 & 8.78 & $36-68$ \\
\hline Baseline number repetition & 63.86 & 6.23 & $53-76$ \\
\hline
\end{tabular}

Means were calculated across the 22 scan acquisitions (two per subject) for each of the five tasks after data were digitally analyzed. Means represent the number of seconds when speech output occurred during the total $90 \mathrm{sec}$ of scan acquisition.

marks selected in common across subjects as those they could visualize but had not visited in person were selected. Landmarks that had not been visited by any subject were selected to prevent subjects from recalling landmark locations within a spatially extended environment, thus assessing the neural basis of the landmarks per se as the most primary elements of topographical knowledge. During each scan, one stimulus item was presented (i.e., one route/film plot/film frame/landmark) for subjects to respond to; this was determined by a pilot study with nonparticipating taxi drivers. Thus, during scanning, subjects engaged in the retrieval tasks throughout the critical window in the rising phase of brain radioactivity. A pilot study outside the scanner using healthy normal volunteers performing the same tasks indicated no differences in electrooculography across the different tasks. The tasks in this study were designed to examine those processes normally engaged in in the real world; as a result, the tasks naturally are complex, with many components. The current stimuli and study design were selected as those best suited to answer the questions posed and to minimize the effect of factors others than those of interest, although there may be other differences between the tasks in addition to those of primary concern.

\section{RESULTS}

\section{Behavioral data}

Table 1 shows the mean duration of speech for each of the tasks that occurred over the $90 \mathrm{sec}$ duration of scan acquisition. Statistical analyses showed no significant differences in speech duration between any of the main tasks. Not surprisingly, given the repetitive nature of the task, significantly more speech occurred during the baseline number repetition task compared with each of the main tasks. The content of speech was also examined. Before arrival for scanning it had been established by questionnaire which stimuli subjects were familiar with, and this was reflected in the high accuracy of subjects' recall. The goal of the study was to engage subjects in the relevant task for the duration of each scan. All subjects performed the tasks fluently, without stopping.

It was notable that the routes chosen by subjects during the navigation task were all very similar, with small differences in the minor roads selected. Street names were recalled accurately, as were relevant landmarks, and traffic restrictions were adhered to. Figure 2 presents an example of the speech output of a taxi driver during one of the navigation scans; the map is included to help illustrate the route taken. Subjects did not actually see maps at any time during the study. In some cases, the names of streets were not recalled, although their spatial location was (e.g., "... take the second street on the left..."); in such cases, this was regarded as correct topographical information. Indeed, even if no street names had been provided in the presence of correct location information this would have been acceptable; accurate verbal labeling was not a prerequisite for accurate topographical knowledge. Subjects were debriefed after scanning and reported visualizing actual travel along the routes, including "seeing" salient landmarks as they were passed. During the tasks describing film plots and frames and famous world landmarks, subjects reported that they recalled the films/frames/landmarks from memory as if they were looking at them. The film frames were described as if in "freeze frame," with no mention of sequential plot action but merely descriptions of who was in the scene and other visual information. Rich descriptions of landmarks were provided, with many details recalled. Overall, subjects denied recalling any specific spatiotemporal context related to any of the information retrieved.

\section{PET results}

Overview

The design of this experiment had two major features: (1) factorial, with two factors of interest (memory type: topographical/ nontopographical; sequencing: with or without), and (2) an additional baseline condition. Analyses based on the factorial design and those based on comparison with the baseline offer complementary insights into the data. Only those analyses germane to the outlined research questions are presented. Comparison with the baseline gave an overall picture of the neural systems supporting each task individually. From this, one can also observe brain regions that tasks activate in common or differently. More direct comparisons between tasks were made within the framework of the factorial design: to determine (1) simple main effects within memory type, (2) compound main effects across memory type and across the sequencing factor, and also to examine (3) the interaction between memory type and sequencing.

\section{Comparisons with baseline}

The relative $\mathrm{rCBF}$ increases associated with the main memory tasks in comparison with the baseline task are presented in Table 2. Comparison of the activity during the routes task with the baseline revealed bilateral activity in extrastriate regions, the medial parietal lobe, the posterior cingulate cortex, and parahippocampal gyrus, and also activation of the right hippocampus. There was no significant activation of frontal regions associated with this comparison. The landmarks task compared with baseline also resulted in activation of the posterior cingulate cortex, the medial parietal lobe, and occipitotemporal regions, including the parahippocampal gyrus. In this case, however, there was no activation of the hippocampus, but there was significant activation of left inferior and middle frontal gyri. In comparison with the baseline, the film tasks (both plots and frames) activated left frontal regions, middle temporal gyrus (plots left, frames right), and left angular gyrus but not medial temporal areas. The cerebellum was activated by all task comparisons with the baseline as was the left temporal pole (subthreshold for the routes comparison). In summary, the answer to our first question is that the network of brain regions, including the right hippocampus, that supports semantic topographical memory retrieval is similar to that noted in a previous study (Maguire et al., 1996a): it supports both the learning of new complex spatial layouts as well as the retrieval of recently acquired topographical memories. The answer to our second question, specifically inquiring about the neural instantiation of landmarks, is that both retrieval of landmark knowledge and retrieval of complex route information activate many similar brain regions, but the right hippocampus is activated only during routes recall.

\section{Simple main effects}

Comparison of the activity during routes recall with activity during any of the other conditions revealed significantly increased activation of the right hippocampus. The two topographical tasks, routes and landmarks, were compared; the 


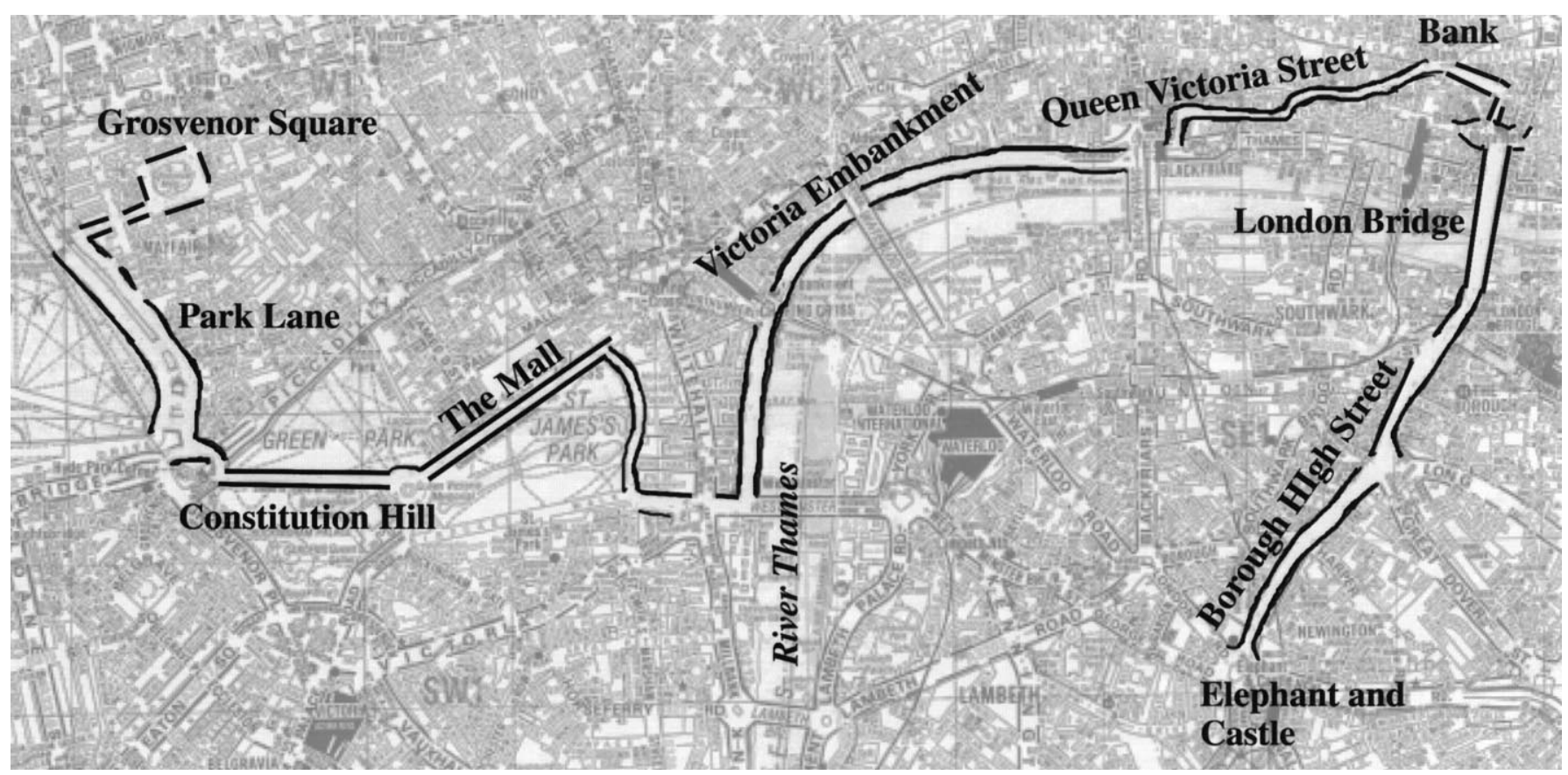

Figure 2. Map illustrating the complex route recalled by a taxi driver during a route scan. Subjects did not see any maps; they were blindfolded throughout. His speech output for this task follows: Pick up on Grosvenor Square in Mayfair, drop off at Bank Underground Station, then at the Oval Cricket Ground. . " Grosvenor square, I'd leave that by Upper Grosvenor Street and turn left into Park Lane. I would eh enter Hyde Park Corner, a one-way system and turn second left into Constitution Hill. I'd enter Queen Victoria Memorial one-way system and eh leave by the Mall. Turn right Birdcage Walk, sorry right Horse Guards Parade, left Birdcage Walk, left forward Great George Street, forward into Parliament Square, forward Bridge Street. I would then go left into the eh the Victoria Embankment, forward the Victoria Embankment under the Blackfriars underpass and turn immediate left into Puddledock, right into Queen Victoria Street, left into Friday Street, right into Queen Victoria Street eh and drop the passenger at the Bank where I would then leave the Bank by Lombard Street, forward King William Street eh and forward London Bridge. I would cross the River Thames and London Bridge and go forward into Borough High Street. I would go down Borough High Street into Newington Causeway and then I would reach the Elephant and Castle where I would go around the one-way system...." (end of scan).

activity during the landmarks task was subtracted from the activity during the routes task. This comparison revealed increased activation of medial parietal cortex, posterior cingulate cortex, and the right hippocampus for routes relative to landmarks. Figure 3 shows this comparison where the activation is superimposed at the level of the hippocampal activation onto the averaged MRI scan of the 11 taxi drivers normalized to the same stereotactic space. This confirms that activity in the right hippocampus is increased during routes recall, as in topographical encoding (Maguire et al., 1996a), but is not increased during the recall of landmarks. The opposite comparison (landmarks vs routes) showed activation in the left inferior frontal gyrus for landmarks compared with routes. Film plots compared with film frames showed no significant changes in rCBF.

\section{Compound main effects}

Comparison of each task with the baseline (Table 2) suggests that very different networks of brain regions were involved in the routes and landmarks tasks on the one hand and the film tasks on the other. The main effect of memory type (topographical vs nontopographical) was determined directly by comparing the activity during topographical tasks (routes and landmarks) with activity during nontopographical tasks (film plots and film frames). This comparison is shown in Figure $4 a$. There was increased activation of the bilateral medial parietal regions, the posterior cingulate cortices, fusiform gyri, and parahippocampal gyri during topographical relative to nontopographical memory retrieval. In summary, the answer to our third question is that the network of brain regions showing increased activation during semantic topographical memory retrieval are entirely different from those activated during retrieval of nontopographical semantic memory. The main effect of sequencing was determined by comparing the activity during tasks involving sequencing (routes and film plots) with activity during nonsequencing tasks (landmarks and film frames). This comparison is shown in Figure $4 b$. The effect of sequencing was shown to be in bilateral medial parietal regions. There was no change of hippocampal activity in relation to sequencing.

\section{Interaction}

Examination of the interaction between memory type (topographical vs nontopographical) and sequencing reveals an interaction in the medial parietal region and in the right inferior parietal cortex (BA 40), where activation is greatest in topographical memory that involves sequencing, as in the recall of routes (Fig. 5).

\section{DISCUSSION}

\section{Retrieval of route knowledge}

The aim of the present study was to examine semantic topographical memory retrieval and to determine whether recall of well established spatial layouts activates similar brain regions noted in other studies to subserve episodic topographical memory, with reference in particular to the medial temporal region. Activation of the parahippocampal gyrus, posterior cingulate cortex, precuneus, and cerebellum has been associated with episodic topo- 
Table 2. Increases in rCBF associated with comparison of each task with the baseline task

\begin{tabular}{|c|c|c|c|c|}
\hline & $\begin{array}{l}\text { Routes } \\
\text { (coordinates } / z \text { score) }\end{array}$ & $\begin{array}{l}\text { Landmarks } \\
\text { (coordinates } / z \text { score) }\end{array}$ & $\begin{array}{l}\text { Film plots } \\
\text { (coordinates/z score) }\end{array}$ & $\begin{array}{l}\text { Film frames } \\
\text { (coordinates } / z \text { score) }\end{array}$ \\
\hline R H & $16,-38,0 / 3.66$ & & & \\
\hline L PHG & $-28,-42,-6 / 4.92$ & $-28,-42,-6 / 4.24$ & & \\
\hline R PHG & $18,-38,-6 / 3.97$ & $(8,-42,0 / 2.90)$ & & \\
\hline L O-T (BA 37) & & $-22,-42,-12 / 4.63$ & & \\
\hline L PCG (BA 31) & $-14,-60,26 / 5.90$ & & & \\
\hline R PCG (BA 30/31) & & $14,-52,20 / 4.48$ & & \\
\hline L SPL (BA 7) & $-20,-70,56 / 5.40$ & & & \\
\hline L AG (BA 39) & & & $-54,-62,18 / 4.03$ & $-54,-62,18 / 3.65$ \\
\hline R MP & $6,-66,62 / 5.00$ & & & \\
\hline L MP & $-10,-66,40 / 4.60$ & $-2,-64,58 / 3.86$ & & \\
\hline L TP (BA 38) & $(-40,18,-26 / 3.00)$ & $-32,22,-34 / 3.68$ & $-36,22,-32 / 4.18$ & $-34,24,-28 / 3.89$ \\
\hline L ITG (BA 20) & & $-40,-30,-22 / 4.55$ & & \\
\hline L MTG (BA 21) & & & $-46,-44,-4 / 3.54$ & $-50,0,-26 / 3.54$ \\
\hline L IFG (BA 44) & & $-50,18,22 / 4.70$ & $-56,24,16 / 3.40$ & \\
\hline L MFG (BA 11) & & $-24,30,-18 / 4.22$ & & \\
\hline L SFG (BA 6) & & $-18,12,52 / 4.08$ & & $-12,10,64 / 3.35$ \\
\hline R SOG (BA 19) & $40,-80,28 / 7.05$ & & & \\
\hline L SOG (BA 19) & $-32,-82,36 / 6.70$ & $-30,-88,32 / 4.11$ & & \\
\hline $\mathrm{L} \mathrm{C}$ & $-48,-78,-24 / 3.87$ & $-14,-42,-16 / 4.38$ & $-8,-86,-40 / 3.52$ & \\
\hline R C & & $34,-76,-40 / 4.50$ & $36,-78,-50 / 4.55$ & $34,-76,-50 / 4.46$ \\
\hline
\end{tabular}

Coordinates in stereotactic space (Talairach and Tournoux, 1988) refer to local maxima. BA, Brodmann's area; R, right; L, left; H, hippocampus; PHG, parahippocampal gyrus; O-T, occipito-temporal; PCG, posterior cingulate gyrus; SPL, superior parietal lobe; AG, angular gyrus; MP, medial parietal (precuneus); TP, temporal pole; ITG, inferior temporal gyrus; MTG, middle temporal gyrus; IFG, inferior frontal gyrus; MFG, middle frontal gyrus; SFG, superior frontal gyrus; SOG, superior occipital gyrus; $\mathrm{C}$, cerebellum. $P<0.001$ (uncorrected) except for those in parentheses, which just failed to reach significance at this level but are included for the sake of completeness.

$$
z=-8 \text { irimin }
$$

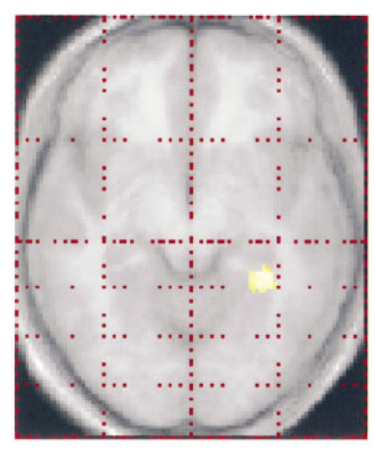

$$
z=-6 \text { mirm }
$$

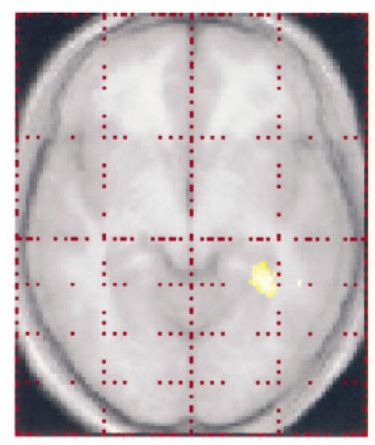

$z=-4 m m i n$

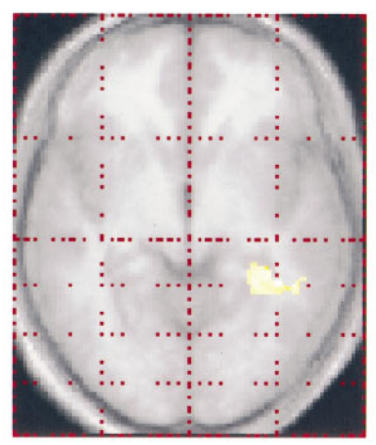

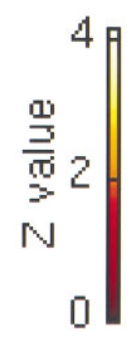

Figure 3. Comparison of routes recall with landmarks recall. The activations are superimposed onto the averaged MRI scan of the 11 taxi drivers normalized to the same stereotactic space. The voxel of peak activation in the right hippocampus has been located here on relevant transverse sections. Other areas of significant activation in this comparison included the medial parietal region and the posterior cingulate gyrus. graphical memory retrieval (Aguirre et al., 1996; Ghaem et al., 1996) and learning of spatially extended environments (Maguire et al., 1996a, 1997). Recall of routes around London by taxi drivers in the present study activated the same brain regions compared with a baseline task. Therefore, it seems that both learning and recall from topographical episodic or semantic memory have broadly the same network of brain regions as their neural substrate. Thus, for topographical memory at least, the distinction between episodic and semantic memory seems to have no anatomical basis.

Our study reveals activation of the hippocampus proper in topographical memory retrieval. This is consistent with animal work that documents the importance of the hippocampus in allocentric mapping of space (O'Keefe and Nadel, 1978). This observation indicates a role for the right hippocampus in processing spatial layouts over long time courses not assessed by the short time scales of previous studies. This study and that of Maguire et al. (1996a), in which activation of the hippocampus proper during topographical learning was also recorded, contrast with the two other topographical memory studies (Aguirre et al., 1996; Maguire et al., 1997) in which hippocampal activation was not found. These studies used computer-simulated environments, whereas the present study and that of Maguire et al. (1996a) involved memory for real-world environments. Real environments are more complex than the simulations used to date and involve the potential for using many routes to navigate to a goal, as reflected in the task put before the taxi drivers to find the shortest route to a destination. The recruitment of the hippocampus when realworld environments are involved may reflect its role in higher level spatial manipulation and decision making. The absence of activation of the hippocampus in the two simulation studies that used novel stimuli (Aguirre et al., 1996; Maguire et al., 1997) and the fact that taxi drivers were familiar with the routes in the present study make it unlikely that hippocampus activation in topographical memory tasks is attributable to novelty of stimuli (Tulving et al., 1994). 
A
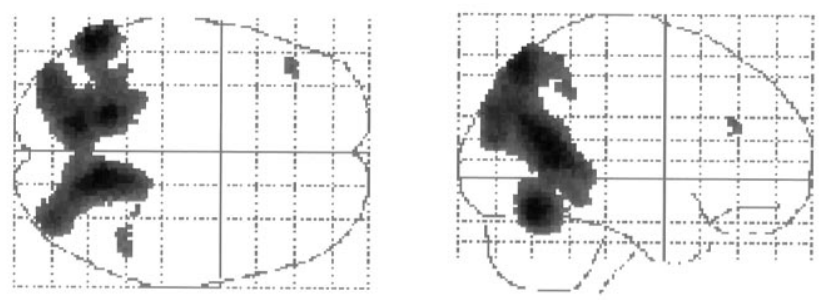

B

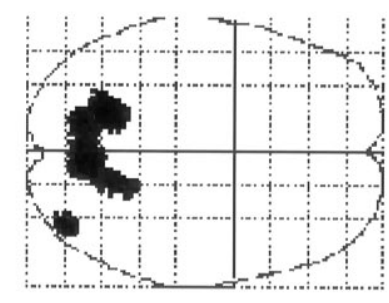

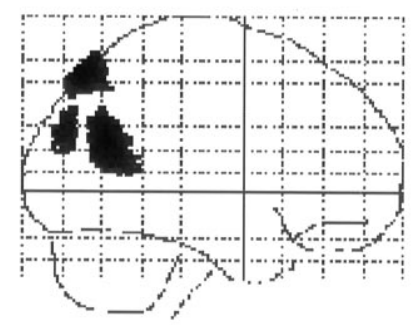

Figure 4. A, Main effect of memory type displayed on a glass brain to show all areas of significant activation. Areas of increased rCBF during topographical memory compared with nontopographical memory. $B$, Main effect of sequencing displayed on a glass brain. Areas of increased activity when sequencing was required for correct memory recall are compared with memory recall for which no particular sequence of information was necessary.

\section{Retrieval of landmark knowledge}

The current study also set out to assess the neural instantiation of landmark knowledge where such knowledge was not confounded by location information about position within a large-scale spatial layout. Both landmarks and routes activated occipitotemporal regions, posterior cingulate gyrus, medial parietal area, and the parahippocampal gyrus in comparison with the baseline. The involvement of many of the same brain regions, both dorsal and ventral, in routes and landmarks memory indicates that the topographical memory system is primed for relevant topographical information even when the landmarks, as in this case, have no spatial connotation. Farrell (1996), in a recent review of topographical disorientation, concluded from the literature that there was no evidence for distinct mechanisms for the identification of environmental features on the one hand and their location on the other. Rather, he concludes that these are two aspects of the same allocentric system located in the ventral stream. Our findings support this view. The main difference between activation patterns for routes and landmarks was that the right hippocampus was activated only in the routes task but not during recall of landmarks. The landmarks lacked a location within a large-scale spatial framework and thoughts of navigation between them were not possible, again suggesting a role for the right hippocampus in the crucial complex stage of facilitating navigation in large-scale space.

The recall of landmarks, but not the recall of routes, was associated with activation of the left lateral prefrontal cortex. Activation of left frontal regions has been noted in many PET studies of memory, most often with verbal episodic memory encoding (Kapur et al., 1994; Shallice et al., 1994). Perhaps the recall of landmarks from semantic memory involves, as Nyberg et al. (1996) suggest, the encoding of such material into (verbal) episodic memory; however, it is not clear why this would be the case for landmarks specifically, but not for recall of routes from semantic memory. Fletcher et al. (1996) suggest that the activation of the left prefrontal cortex associated with cued recall of nonimageable words may reflect an attempt by subjects to form

alternative semantic links between cue and response not necessary when words are highly imageable. Landmarks are usually very important anchor points in the representation of spatially extended environments. One might speculate that recall of landmarks that are devoid of position within a spatial layout may invoke functions and areas not normally concerned with the representation of space when it is within a coherent framework.

\section{Semantic memory type}

A final aim of the study was to examine topographical memory (landmarks/spatial layouts) and also nontopographic semantic memory retrieval to ascertain whether common brain regions subserve semantic memory regardless of memory type. The use of familiar film plots as stimuli engaged subjects in memory recall at a similar level and with broadly similar characteristics as in the routes task, such as the recall of information in a specific temporal sequence (i.e., progression along a route or progression of a story line). Except for cerebellar activity, recall of film plots was associated with brain regions different from those activated during the routes task. Most activity was left-sided, and there was no activation of occipitotemporal or medial temporal regions when compared with the baseline. The recall of film plots and frames, however, resulted in activation of the left inferior frontal gyrus. All of the semantic memory recall tasks activated the left inferior frontal region, except for the routes recall task. Therefore, it may be the case that this activation is associated, as Nyberg et al. (1996) suggest, with the encoding of semantic knowledge (including spatially devoid landmarks) back into episodic memory, or perhaps this area houses a mechanism common to both episodic memory encoding and semantic memory retrieval. Topographical memory for large-scale space, however, is clearly not recalled via this mechanism, and seems to remain a function of the posterior brain. It might be argued that the taxi drivers, who were very knowledgeable about the spatial layout of London, were so practiced that their responses were automatic and so required no increased activation of frontal regions; however, the task required them to plan the shortest routes between start and destination points, and analysis of verbal output clearly demonstrated that subjects reflected on their responses in a considered and nonautomatic manner.

Case reports in which topographical disorientation is the primary deficit are most consistently reported after posterior brain lesions. The ability to navigate in large-scale space is one that humans share with a multitude of other species. Many species with a smaller relative area of prefrontal cortex than that of humans are able to navigate successfully, suggesting that perhaps other more posterior brain areas are most involved in such abilities. Other work has found that it is the size of the hippocampus relative to the size of the telencephalon that varies according to whether spatial skills are critical to survival. For example, in food-storing birds, the hippocampus is larger than in species who cache food to a lesser extent (Sherry et al., 1992; Hampton et al., 1995). Topographical memory is a phylogenetically old ability and perhaps depends less on frontal regions, the hippocampal region being sufficient for its support. Of course, it is possible that frontal regions are recruited into topographical memory processing under circumstances that have not yet been examined in functional imaging studies, although notably, frontal regions were not activated in two previous PET studies of topographical learning (Maguire et al., 1996a, 1997).

The medial parietal region (precuneus) has been activated in many PET memory studies and is often held to be associated 


$$
z=48 \mathrm{~mm}
$$

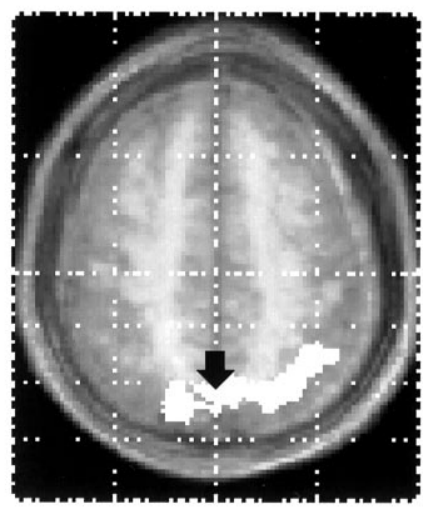

$z=50 \mathrm{~mm}$

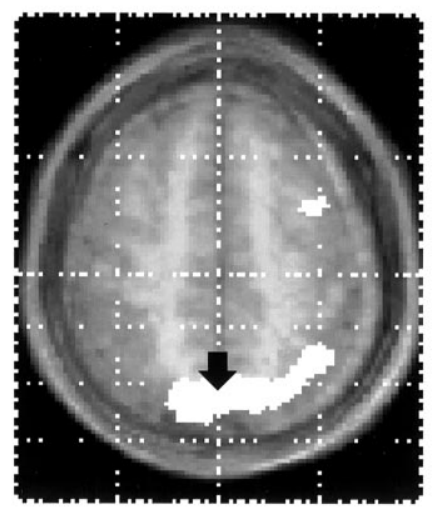

$z=52 \mathrm{~min}$

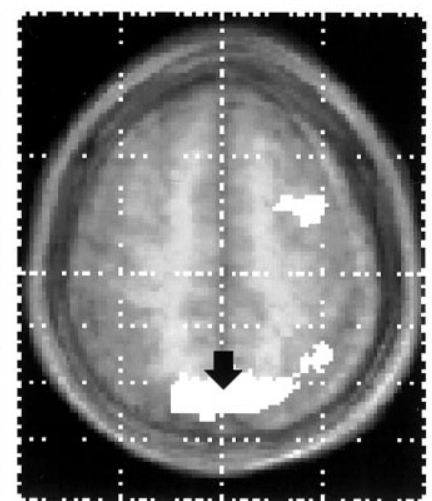

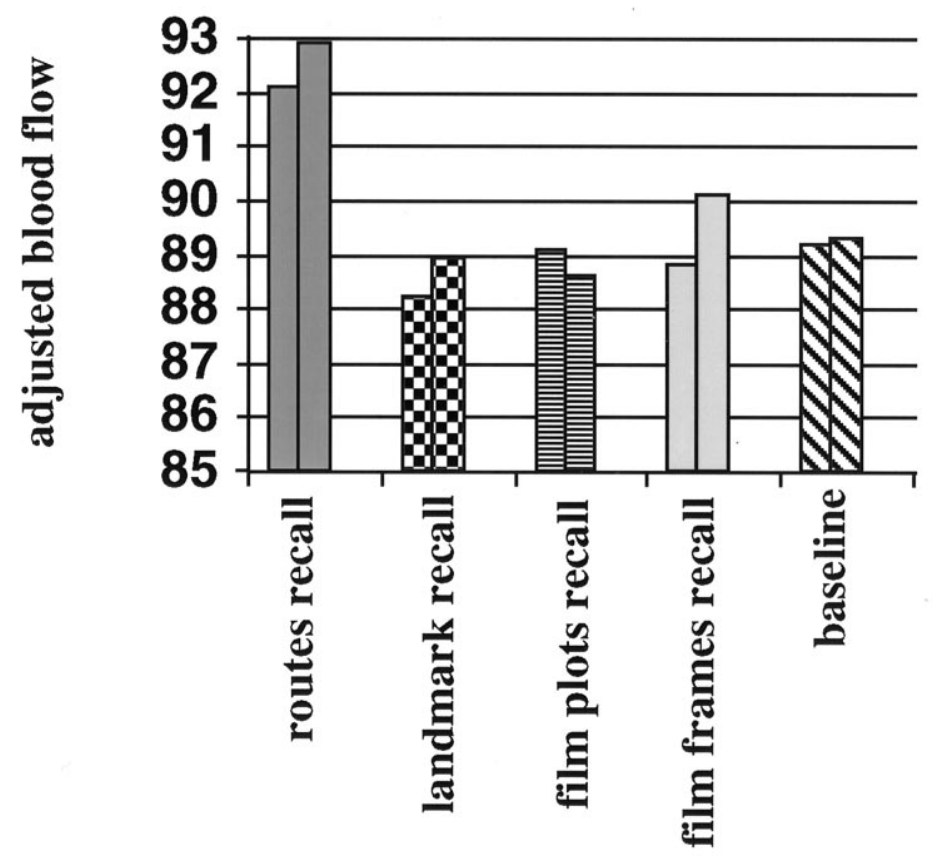

Figure 5. Brain areas in which the effects of memory type and sequencing interacted are shown here on relevant transverse sections of the averaged MRI scan of the 11 taxi drivers. The interaction was determined by the following comparison [(routes-landmarks) - (film plots-film frames)]. The arrow indicates the voxel of peak activation in the medial parietal region; the graph (bottom) shows the adjusted blood flow values at this voxel, revealing that the increased activity in this area is attributable primarily to sequencing in topographical memory (the two histogram bars for each task represent the two scans of the total 12 scans during which this task was performed). with visual imagery (Fletcher et al., 1995a). This is a function noted previously (Maguire et al., 1997) to be compatible with the requirements of a topographical memory system. The findings of the present study, however, throw more light on the function of the parietal region in the context of topographical memory. A significant feature of both film story lines and navigation is their inherent sequential nature; for example, to go from a to $b$ one might have to go via $x, y$, and $z$. It would seem that the medial and right inferior parietal cortex, and not the hippocampal region, are particularly involved in such sequencing, with the interaction analysis showing that this is particularly the case in the context of topographical memory. A significant feature of person-centered or egocentric spatial processing is its sequential nature; this compares to the greater flexibility of more map-like object-centered or allocentric spatial representations. The parietal cortex has been proposed to be involved in egocentric aspects of topographical orientation (O'Keefe and Nadel, 1978; Gross and Graziano, 1995). Our finding that medial and inferior parietal cortex activation is specifically modulated by sequencing requirements in topographical memory is further support for this view.

In summary, the network of brain regions subserving topographical memory in humans, whether during encoding or retrieval of recent or well established topographical knowledge, includes occipitotemporal areas, medial parietal cortex, posterior cingulate cortex, and the parahippocampal gyrus. The right hippocampus is recruited, over long and short time courses, when the environments involved are complex, as they are in the real world, offering multiple routes to a destination. The brain system for topographic representation is undiscriminating and flexible, activating in the presence of any kind of topographic stimulation. In contrast, semantic memory for nontopographic information involves increased activity in the left inferior frontal gyrus but not in the hippocampus. Topographical memory retrieval does not engage this frontal region and seems to remain a function of the posterior brain. 


\section{REFERENCES}

Aguirre GK, Detre JA, Alsop DC, D’Esposito M (1996) The parahippocampus subserves topographical learning in man. Cereb Cortex 6:823-829.

Chase WG (1983) Spatial representation of taxi drivers. In: The acquisition of symbolic skill (Rogers D, Sloboda JA, ed), pp 391-405. New York: Plenum.

DeRenzi E, Faglioni P, Villa P (1977) Topographical amnesia. J Neurol Neurosurg Psychiatry 40:498-505.

Evans AC, Collins DL, Mills SR, Brown ED, Kelly, RL, Peters TM (1993) 3D statistical neuroanatomical models from 305 MRI volumes. In: Proceedings of the IEEE-Nuclear Science Symposium and Medical Imaging Conference, pp 1813-1817.

Farrell MJ (1996) Topographical disorientation. Neurocase 2:509-520.

Fletcher PC, Frith CD, Baker SC, Shallice T, Frackowiak RS, Dolan RJ (1995a) The mind's eye: precuneus activation in memory-related imagery. NeuroImage 2:195-200.

Fletcher PC, Frith CD, Grasby PM, Shallice T, Frackowiak RSJ, Dolan RJ (1995b) Brain systems for encoding and retrieval of auditory-verbal memory. Brain 118:401-416.

Fletcher PC, Shallice T, Frith CD, Frackowiak RSJ, Dolan RJ (1996) Brain activity during memory retrieval. The influence of imagery and semantic cueing. Brain 119:1587-1596.

Friston KJ, Frith CD, Liddle PF, Frackowiak RSJ (1991) Comparing functional (PET) images: the assessment of significant change. J Cereb Blood Flow Metab 11:690-699.

Friston KJ, Worsley KJ, Frackowiak RSJ, Mazziotta JC, Evans AC (1994) Assessing the significance of focal activations using their spatial extent. Hum Brain Mapp 1:214-220.

Friston KJ, Holmes A, Worsley KJ, Poline JB, Frith CD, Frackowiak RSJ (1995) Statistical parametric maps in functional imaging. A general linear approach. Hum Brain Mapp 2:189-210.

Ghaem O, Mellet E, Tzourio N, Joilot M, Crivello F, Petit L, Laurier L, Berthoz A, Mazoyer B, Denis M (1996) Functional anatomy of mental simulation of memorised routes. NeuroImage 3:S249.

Giraudo M-D, Peruch P (1988) Spatio-temporal aspects of mental representation of urban space. J Environ Psychol 8:9-17.

Gross CG, Graziano MSA (1995) Multiple representations of space in the brain. Neuroscientist 1:43-50.

Habib M, Sirigu A (1987) Pure topographical disorientation: a definition and anatomical basis. Cortex 23:73-85.

Hampton RR, Sherry DF, Shettleworth SJ, Khurgel M, Ivy G (1995) Hippocampal volume and food-storing behavior are related in parids Brain Behav Evol 45:54-61.

Kapur S, Craik FIM, Tulving E, Wilson AA, Houle S, Brown GM (1994)
Neuroanatomical correlates of encoding in episodic memory: levels of processing effect. Proc Natl Acad Sci USA 91:2008-2011.

Landis T, Cummings JL, Benson DF, Palmer EP (1986) Loss of topographical familiarity: an environmental agnosia. Arch Neurol 43:132-136.

Maguire EA, Frackowiak RSJ, Frith CD (1996a) Learning to find your way: a role for the human hippocampal region. Proc R Soc Lond [Biol] 263:1745-1750.

Maguire EA, Burke T, Phillips J, Staunton H (1996b) Topographical disorientation following unilateral temporal lobe lesions in humans. Neuropsychologia 34:993-1001.

Maguire EA, Burgess N, Donnett JG, O'Keefe J, Frith CD (1997) Knowing where things are: parahippocampal involvement in encoding object locations in virtual large-scale space. J Cognit Neurosci, in press.

Nyberg L, Cabeza R, Tulving E (1996) PET studies of encoding and retrieval. Psychonomic Bull Rev 3:135-148.

O'Keefe J, Nadel L (1978) The hippocampus as a cognitive map. Oxford: Clarendon.

Pailhous J (1984) The representation of urban space: its development and its role in the organisation of journeys. In: Social representations (Farr RM, Moscovici S, eds), pp 311-396. Cambridge, England: Cambridge UP.

Peruch P, Giraudo M-D, Garling T (1989) Distance cognition by taxi drivers and the general public. J Environ Psychol 9:233-239.

Shallice T, Fletcher PC, Frith CD, Grasby P, Frackowiak RSJ, Dolan RJ (1994) Brain regions associated with acquisition and retrieval of verbal episodic memory. Nature 368:633-635.

Sherry DF, Jacobs LF, Gaulin SJ (1992) Spatial memory and adaptive specialization of the hippocampus. Trends Neurosci 15:298-303.

Siegal AW, White SH (1975) The development of spatial representation of large-scale environments. In: Advances in child development and behavior, Vol 10 (Reese HW, ed), pp 9-55. New York: Academic.

Talairach J, Tournoux P (1988) Co-planar stereotactic atlas of the human brain. Stuttgart, Germany: Thieme.

Thorndyke PW, Hayes RB (1982) Difference in spatial knowledge acquired from maps and navigation. Cognit Psychol 14:560-589.

Tulving E (1983) Elements of episodic memory. Oxford: Clarendon.

Tulving E, Markowitsch HJ, Kapur S, Habib R, Houle S (1994) Novelty encoding networks in the human brain: positron emission tomography data. NeuroReport 5:2525-2528.

Tulving E, Markowitsch HJ, Craik FIM, Habib R, Houle S (1996) Novelty and familiarity activations in PET studies of memory encoding and retrieval. Cereb Cortex 6:71-79.

Worsley KJ, Evans AC, Marrett S, Neelin P (1992) A three-dimensional statistical analysis for $\mathrm{rCBF}$ activation studies in human brain. J Cereb Blood Flow Metab 12:900-918. 